\title{
Exotic grain growth law in twinned boron carbide under electric fields
}

\author{
Bibi Malmal Moshtaghioun*1,2, Diego Gómez-García ${ }^{1,3}$, Arturo Domínguez Rodríguez ${ }^{1}$ \\ ${ }^{1}$ Departamento de Física de la Materia Condensada, Universidad de Sevilla, P. O. 1065, 41080 \\ Sevilla, Spain. \\ ${ }^{2}$ Instituto de Ciencia de Materiales de Aragón, CSIC-Universidad de Zaragoza, campus Río \\ Ebro, Sede Campus Río Ebro, Edificio Torres Quevedo, calle María de Luna 3, 50018 Zaragoza, \\ Spain. \\ ${ }^{3}$ Instituto de Ciencia de Materiales de Sevilla, Centro Mixto CSIC-US, Avenida Americo \\ Vespucio 11, 41092, Sevilla, Spain.
}

\begin{abstract}
*Corresponding author: Bibi Malmal Moshtaghioun, Department of Condensed Matter Physics, Universidad de Sevilla, P. O. Box 1065, 41080 Sevilla, Spain; Tel: +349559504, e-mail addresses: mali_moshtagh@us.es, mali@unizar.es. Now in sabbatical leave at the Instituto de Ciencia de Materiales de Aragón, Universidad de Zaragoza, campus Río Ebro, Zaragoza, Spain.
\end{abstract}

\begin{abstract}
Grain growth is a ubiquitous phenomenon in all materials, and it affects both structural and functional properties. Despite its intrinsic importance, a full comprehension of grain growth from a fundamental point of view-i.e., from the nanoscale to the macroscale - is still a pending issue. In practical terms, our knowledge relies on the classical kinetic laws reported sixty years ago.
\end{abstract}

This paper reports the violation of such classical laws in boron carbide ceramics consolidated by spark plasma sintering. The conjunction of high temperature gradients with large 
https://daneshyari.com/article/7897731 